\title{
Vida acadêmica e exploração vocacional em universitários formandos: relações e diferenças
}

\section{Vocational exploration and academic life in college students: relationships and differences}

\section{Vida académica y la Exploración vocacional en los estudiantes universitarios: relaciones y diferencias}

\author{
J ocemara Ferreira Mognon* \\ Universidade São Francisco - USF, I tatiba, São Paulo, Brasil \\ Acácia Aparecida Angeli dos Santos** \\ Universidade São Francisco - USF, I tatiba, São Paulo, Brasil
}

\begin{abstract}
RESUMO
O objetivo deste estudo foi verificar a relação entre vida acadêmica e exploração vocacional, bem como possíveis diferenças em razão das variáveis sexo, idade e curso. Participaram 213 formandos que frequentavam diversos cursos em uma universidade particular do interior do estado de São Paulo, com idades entre 21 a 57 anos ( $M=24,62$ anos), sendo $64,8 \%$ do sexo masculino. Foram utilizadas a Escala de Avaliação da Vida Acadêmica (EAVA) e a Escala de Exploração Vocacional (EEV). Os resultados indicaram correlações significativas e positivas entre os fatores da EAVA e as subescalas da EEV. Foram encontradas diferenças estatisticamente significativas com maiores médias para as mulheres nos dois instrumentos, para os estudantes da faixa etária dos 21 a 23 anos na EAVA, para o curso de Fisioterapia na EAVA e para Psicologia na EEV. Conclui-se que a universidade pode contribuir com a exploração vocacional, mas faz-se necessário considerar as características pessoais dos estudantes.
\end{abstract}

Palavras-chave: ensino superior, planejamento de carreira, desenvolvimento profissional.

\begin{abstract}
The aim of this study was to investigate the relationship between academic life and career exploration, as well as possible differences due to gender, age and course. It took part 213 trainees which were attending various courses at a private university in the state of São Paulo, aged from 21 to 57 years ( $M=24.62$ years), with $64.8 \%$ male. It was used the Scale for Assessment of Academic Life (EAVA) and the Scale of Vocational Exploration (EEV). The results showed significant and positive correlations between the factors of EAVA and the subscales of EEV. It was found statistically significant differences with higher averages for women in the two instruments, for students of the age group of 21-23 years in EAVA, for the course of Physiotherapy in EAVA and Psychology in EEV. It is concluded that
\end{abstract}


the university can contribute to vocational exploration, but it is necessary to consider the personal characteristics of the students.

Keywords: higher education, career planning, professional development.

\begin{abstract}
RESUMEN
El objetivo de este estudio fue investigar la relación entre la vida académica y la exploración de carreras, así como las posibles diferencias por sexo, edad y curso. Participaron 213 estudiantes de diversos cursos en una universidad privada en el estado de São Paulo, con edades entre 21 a 57 años ( $M=24,62$ años), siendo el 64,8 \% hombres. Los instrumentos fueron la Escala de Evaluación de la Vida Académica (EAVA) y la Escala de Exploración Vocacional (EEV). Los resultados mostraron una correlación significativa y positiva entre los factores de la EAVA y las subescalas EEV. Las diferencias fueron estadísticamente significativas con los promedios más altos para las mujeres en los dos instrumentos, para que los estudiantes del grupo de edad de 21-23 años en EAVA, para el curso de Fisioterapia en EAVA y Psicología en EEV. En conclusión, la universidad puede contribuir a la exploración vocacional, pero es necesario tener en cuenta las características personales de los estudiantes.
\end{abstract}

Palabras clave: educación superior, planificación de la carrera, desarrollo profesional.

\title{
1 Introdução
}

Para muitos estudantes a entrada na universidade corresponde à concretização de um sonho (Almeida \& Soares, 2004), que os leva a confrontar-se com novos desafios, visto que a sua adaptação a este novo ambiente dependerá de muitas variáveis tanto pessoais como ambientais (Teixeira, Dias, Wottrich, \& Oliveira, 2008). Dessa forma, a transição para o ensino superior tem sido caracterizada como um evento que pode gerar desgastes, dada as mudanças e o enfrentamento de desafios típicos deste momento (Santos, Polydoro, Bardagi, \& Teixeira, 2010).

Segundo Almeida e Soares (2004), na transição ao ensino superior são importantes não apenas aspectos do meio acadêmico ou de aprendizagem, como também o meio social que impulsiona novas relações interpessoais com professores e colegas. Como ressaltam os autores, a adaptação à vida acadêmica consiste em um processo complexo e multifacetado que é influenciado por fatores tanto de ordem pessoal como também do contexto no qual está inserido. Esse processo de interação entre as variáveis inerentes ao contexto universitário enseja o estudante a modificar o ambiente em razão de suas percepções, escolhas, objetivos, esforços e ações, e ainda, sendo ele mesmo também modificado pelas normas, exigências, expectativas e oportunidades relativas à instituição (Santos et al., 2010).

Além da entrada na universidade, outro momento de muitas expectativas e ansiedade vivenciado pelos estudantes é a formatura. 
Para Teixeira e Gomes (2004), o fim do curso universitário é um momento de transição na busca por estabelecer novos objetivos profissionais e pessoais, com base nas experiências já vivenciadas. No entanto, atualmente, os postos de trabalho exigem atualização profissional constante, bem como capacidade para aprender e de se adaptar (Bardagi \& Boff, 2010; Santos, Mognon, \& Joly, 2011). Com isso, percebe-se um movimento do próprio aluno na construção do seu desenvolvimento profissional, qualificando-se e buscando adquirir competências ao longo da formação acadêmica (Teixeira \& Gomes, 2004).

Considerando-se esses aspectos, verifica-se que há muito o que se investigar sobre a vida acadêmica, com o objetivo de fornecer subsídios para que as Instituições de Ensino Superior (IES) criem condições mais adequadas para adaptação do estudante, favorecendo o seu bem-estar psicológico (Teixeira, Castro, \& Piccolo, 2007). Especificamente para os formandos, as ações das IES devem favorecer a elaboração de projetos de desenvolvimento de carreira a fim de que a transição universidade-mercado de trabalho seja realizada pelo estudante de forma mais consciente e planejada (Santos et al., 2011).

Conforme apontam Santos et al. (2010) apesar da relevância que o conhecimento sobre o estudante do ensino superior tem para a realidade brasileira, não há pesquisas suficientes para avaliar a diversidade dessa experiência de formação acadêmica. Segundo os autores, são restritos os trabalhos que, de fato, apresentam o universitário como objeto central de estudo. No levantamento realizado pelos autores, foram encontrados apenas quatro instrumentos que investigam a vida acadêmica dos universitários, 0 Questionário de Vivência Acadêmica - QVA (Almeida, Soares, \& Ferreira, 2004; Santos, Noronha, Amaro, \& Villar, 2005) e a sua versão reduzida QVA-r (Granado, 2004), a Escala de Integração ao Ensino Superior - EIES (Polydoro, Primi, Serpa, Zenorini, \& Pombal, 2001) e a Escala de Avaliação da Vida Acadêmica - EAVA (Vendramini et al., 2004).

Ainda, segundo Santos et al. (2010) o QVA-r é um dos instrumentos mais utilizados no contexto brasileiro para avaliar a adaptação acadêmica. No entanto, é importante que os estudos avancem também em relação a outras escalas que avaliam as experiências na universidade. Com esse propósito, Lemos (2010) buscou evidências de validade convergente entre a QVA-r e a EAVA, os resultados indicaram correlações positivas, de magnitude baixa a alta, entre todas as dimensões dos instrumentos. Segundo a autora, ficou evidenciado que ambas as escalas avaliam construtos similares. Dentre os diversos resultados encontrados com a EAVA, a autora destaca as diferenças estatisticamente significativas para a variável sexo com maiores médias para as mulheres nos fatores compromisso 
com o curso, condições para o estudo e desempenho acadêmico. $\mathrm{Na}$ comparação que fez entre as etapas do curso (iniciantes, intermediários e concluintes) identificou diferenças estatisticamente significativas, com maiores médias para os iniciantes nos fatores ambiente universitário e compromisso com o curso. Com relação à idade, verificou que os estudantes da faixa etária acima dos 23 anos apresentaram médias estatisticamente maiores nos fatores ambiente universitário, compromisso com o curso, envolvimento em atividades não obrigatórias e, condições para o estudo e desempenho acadêmico.

Outro estudo realizado com a EAVA foi o de Santos, Mognon, Lima e Cunha (2011). As autoras buscaram identificar as relações entre a vida acadêmica e a motivação para aprendizagem, além de possíveis diferenças em razão do sexo, idade e curso dos estudantes. Participaram 239 universitários que utilizaram além da EAVA a Escala de Motivação para Aprendizagem (EMAPRE). Os resultados indicaram que 0 fator habilidade do estudante mostrou-se associado positivamente às metas de realização especificamente voltadas para a valorização da aprendizagem, enquanto que os fatores envolvimento em atividades não obrigatórias e, condições para o estudo e desempenho acadêmico foi negativamente associado a esse tipo de meta. No que se refere especificamente as diferenças para as variáveis de estudo e a EAVA, os resultados indicaram diferenças significativas com maiores médias para as mulheres nos fatores compromisso com o curso, condições para o estudo e desempenho acadêmico. Quanto aos cursos também foram encontradas diferenças estatisticamente significativas, com maiores médias para os alunos que frequentavam Arquitetura no fator ambiente universitário, enquanto que para os de Administração no fator envolvimento em atividades não obrigatórias. Por fim, para a idade não foram encontradas diferenças estatisticamente relevantes.

Até o momento foram encontrados somente estes dois estudos com a EAVA, nos bancos de dados pesquisados Scielo, Bvs-psi e Redalyc. Novamente, verifica-se a escassez de pesquisas no ensino superior atingindo notoriamente alguns instrumentos de avaliação e construtos. No que se refere a este último, por exemplo, está a exploração vocacional. Bardagi e Hutz (2010) também salientam que são poucos os estudos no Brasil que investigam a exploração vocacional nos universitários, visto que as pesquisas sobre essa temática são predominantemente voltadas para os alunos do Ensino Médio.

Para muitos autores (Bardagi, 2005; Pelissoni, 2007; Teixeira et al., 2007) o conceito de comportamento exploratório vocacional designa uma conduta do estudante que possibilita o acesso à informação facilitando o aprendizado, organizando as experiências, proporcionando maturidade para a carreira, autoconhecimento para 
pensar sobre os seus valores, interesses, habilidades e na busca por objetivos futuros. Resumidamente, pode ser considerado um comportamento de solução de problemas em que o objetivo é reunir informações sobre si e o ambiente que facilite ao estudante escolher, preparar-se e progredir no seu campo de trabalho (Gerk, Cardoso, \& Krafft, 2011).

O construto comportamento exploratório vocacional envolve dois aspectos que são a exploração de si e a do ambiente. A primeira relaciona-se com comportamento de busca para conhecer-se a si mesmo, suas próprias capacidades, habilidades e traços de personalidade com respeito à escolha vocacional. Enquanto que a exploração do ambiente envolve a busca de conhecimento da realidade profissional e alternativas educacionais de aprimoramento (Teixeira et al., 2007).

O processo de exploração vocacional pode ser considerado um ponto importante para o desenvolvimento de carreira no estudante. Segundo Bardagi (2005), o desenvolvimento de carreira é um processo de aprendizagem que envolve o crescimento do indivíduo por meio do aperfeiçoamento que ocorre mesmo durante a graduação e também na atuação profissional. Vieira e Coimbra (2006) salientam que a preocupação com o mundo do trabalho não ocorre apenas com o fim do curso, mas, também, durante toda a formação o estudante preocupa-se com esse fator. Mas, Bardagi (2005) ressalta que mesmo que o estudante se depare com escolhas durante a sua vida acadêmica é no período de pré-vestibular e aquele próximo ao término do curso os momentos de maior exploração vocacional.

Alguns estudiosos têm se interessado pela temática, fim do curso universitário e as suas pesquisas têm abordado vários aspectos da passagem da universidade para o mercado de trabalho. A pesquisa de Teixeira e Gomes (2004) é uma delas, visto que buscou explorar as experiências de transição da universidade para o mercado de trabalho em formandos, por meio de entrevistas com roteiro flexível e exploratório sobre aspectos da trajetória na universidade; impressões sobre o mercado de trabalho; preparação profissional recebida e as expectativas para o futuro. Os resultados indicaram que os estudantes têm expectativas otimistas em relação ao seu futuro profissional e um dado importante evidenciou que os universitários que tinham maiores envolvimentos em práticas não obrigatórias eram justamente os que tinham projetos pós-universidade já formulados.

Nesta mesma perspectiva, o estudo de Pelissoni (2007) se propôs a analisar as crenças de autoeficácia na transição para o trabalho e os comportamentos exploratórios em 351 estudantes formandos dos cursos de licenciatura de uma universidade pública. Os instrumentos utilizados foram a Escala de Autoeficácia na Transição para o Trabalho (AETT-Br) e a Escala de Exploração Vocacional (EEV). Os resultados indicaram que, de maneira geral, os estudantes sentiam- 
se confiantes para a passagem universidade - mercado de trabalho e apresentaram altos níveis de comportamentos de exploração vocacional. Em relação à variável sexo, as mulheres apresentaram escores significativamente menores na dimensão autoeficácia na regulação emocional. Em relação ao curso frequentado, somente foram encontradas diferenças significativas na dimensão autoeficácia na adaptação ao trabalho (AETT-Br), sendo que os estudantes da área de exatas obtiveram pontuações superiores. Os resultados indicaram correlações significativas com magnitude fraca a moderada entre as dimensões da $\mathrm{AETT}-\mathrm{Br}$ (autoeficácia na adaptação para o trabalho; autoeficácia na regulação emocional e autoeficácia na procura por emprego) com as duas subescalas da EEV (exploração de si e exploração do ambiente).

Com o propósito de analisar o comportamento exploratório vocacional com outras variáveis potencialmente a ele relacionados, como o autoconceito e a autoeficácia profissional, Bardagi e Boff (2010) avaliaram 231 formandos de diversos cursos de graduação valendo-se de instrumentos de medida específicos, a saber, Escala de Exploração Vocacional (EEV), Escala de Autoconceito e Autoeficácia Profissional. Os resultados indicaram que as mulheres apresentaram níveis mais altos em exploração do ambiente em comparação aos homens e não houve diferenças significativas para a autoeficácia profissional e clareza de autoconceito. Houve diferenças em relação ao curso, em que os alunos da área de saúde declararam maiores dificuldades na elaboração de metas, enquanto os alunos de exatas declararam não perceber dificuldades para a realização do projeto profissional. Os estudantes que possuíam planos pósuniversidade definidos foram os que apresentaram níveis maiores de exploração do ambiente e de autoeficácia profissional.

Outros construtos também associados ao comportamento exploratório vocacional, tais como a satisfação de vida e o comprometimento com o curso foram focalizados por Bardagi e Hutz (2010). Desta feita, os participantes foram 939 universitários de duas instituições públicas e uma privada. Foram utilizadas as Escala de Satisfação de Vida, de Comprometimento de Carreira e a Escala de Exploração Vocacional (EEV). Os resultados indicaram que os alunos sentem-se satisfeitos com o curso e a satisfação apareceu relacionada com o comprometimento com a carreira e com os comportamentos de exploração vocacional. Os alunos que têm planos definidos pós-formatura são os que apresentaram maior comprometimento com o curso e mais comportamentos de exploração. Os resultados indicaram que as atividades acadêmicas relacionam-se com a exploração de si e do ambiente. Não houve diferenças estatisticamente significativas para nenhum construto quanto às variáveis sexo, turno ou tipo de universidade. Mas, quanto ao período do curso, os alunos iniciantes apresentaram maior 
comprometimento com a carreira, mas menor exploração, quando comparados aos formandos.

O estudo de Gerk et al. (2011) analisou as diferenças para a exploração vocacional quanto às variáveis sexo e cursos. Participaram 42 estudantes universitários, com média de idades de 22,3 anos, dos cursos de Psicologia, Direito e Engenharia. O instrumento utilizado foi a Escala de Exploração Vocacional (EEV) e os resultados indicaram que as mulheres obtiveram médias superiores que os homens nas dimensões exploração de si e exploração do ambiente. Observaram ainda, que os alunos de Direito obtiveram maiores médias quando comparados com os da Psicologia e da Engenharia nas dimensões exploração de si e do ambiente da EEV.

Com base nessas considerações, verifica-se a importância do contexto da vida acadêmica no desenvolvimento dos estudantes como facilitadores de comportamentos de exploração vocacional e, consequentemente, para a elaboração de metas e projetos profissionais. Considera-se também, que as variáveis pessoais do próprio estudante têm relevância nesse contexto. Assim, considerando os aspectos focalizados, a presente pesquisa foi proposta com os objetivos de verificar a relação entre a vida acadêmica e a exploração vocacional em estudantes formandos e identificar possíveis diferenças entre eles, considerando as variáveis sexo, idade e curso.

\section{Método}

\subsection{Participantes}

Fizeram parte do estudo 213 estudantes de uma universidade particular do estado de São Paulo, regularmente matriculados no último ano dos cursos, a saber, Fisioterapia $(n=44)$; Engenharia Mecânica $(n=42)$; Engenharia da Computação $(n=33)$; Engenharia Elétrica $(n=34)$; Engenharia Civil $(n=28)$ e Psicologia $(n=32)$. Participaram da pesquisa estudantes de ambos os sexos, sendo $64,8 \%(n=138)$ estudantes do sexo masculino e $35,2 \%(n=75)$ do feminino, com idades entre 21 a 57 anos $(M=24,62$ anos; $D P=7,72)$ e que foram dividas em três grupos, a saber, 'tradicional' de 21 a 23 anos (38,7\%); 'borderlines' de 24 a 26 anos (26,8\%) e 'maduros' acima de 27 anos (34,5\%), conforme classificação adotada por autores como Hoskins, Newstead e Dennis (1997) e Trueman e Hartley (1996). A escolha dos cursos se deu por conveniência, sendo incluídos aqueles em que a instituição disponibilizou para a realização da coleta de dados.

\subsection{I nstrumentos}


Escala de Avaliação da Vida Acadêmica - EAVA - (Vendramini et al., 2004). Tem como objetivo captar a autopercepção dos estudantes sobre a sua vivência universitária por meio de itens que envolvem questões contextuais, interacionais e pessoais. É composta de 34 itens, com cinco fatores. Os itens da escala foram dispostos em uma escala Likert de cinco pontos, sendo: discordo totalmente ( 1 ponto), discordo na maioria das vezes ( 2 pontos), discordo e concordo em igual proporção ( 3 pontos), concordo na maioria das vezes (4 pontos) e concordo totalmente ( 5 pontos). A pontuação mínima possível é de 34 pontos e a máxima de 170 pontos.

A análise psicométrica demonstrou resultados satisfatórios, tendo sido encontrado um índice de consistência interna geral $(a l f a=0,87)$. Os coeficientes e a definição de cada fator são, Ambiente universitário (alfa $=0,72$ - refere-se ao contexto específico, organizado e com características próprias no qual se vincula a ação do estudante, destacando-se o papel desse ambiente, assim como suas características físicas, sociais e organizacionais); Compromisso com o curso (alfa $=0,78$ - refere-se o grau de certeza do estudante em relação à escolha realizada e a percepção de segurança quanto à formação profissional oferecida pelo curso); Habilidade do estudante (alfa $=0,80$ - refere-se ao potencial educacional do indivíduo, considerando-se suas habilidades básicas, escolaridade prévia e condições pessoais); Envolvimento em atividades não obrigatórias (alfa $=0,71$ - diz respeito ao engajamento do aluno em experiências que não se configuram como exigência formal do curso, mas que são promovidas ou incentivadas pela universidade); Condições para o estudo e desempenho acadêmico (alfa $=0,73$ - o desenvolvimento e a aprendizagem do estudante são explicados pelo investimento de energia física e psicológica, e o pelo tempo gasto com as demandas acadêmicas).

Escala de Exploração Vocacional - EEV (Teixeira et al., 2007).

Os itens do instrumento foram criados a partir das definições de exploração vocacional de si (comportamentos refletem autoconhecimento sobre características de personalidade, interesses, habilidades, valores e influências relacionadas à escolha ocupacional) e exploração vocacional do ambiente (comportamentos que visam obter informações das atribuições do seu curso sobre o mundo do trabalho). A versão final da escala ficou com 26 itens. Para as respostas é utilizada uma escala do tipo Likert de cinco pontos, com as seguintes opções: 1 - Raramente ou nunca; 2 - Poucas vezes; 3 Com alguma freqüência; 4 - Freqüentemente; 5 - Muito freqüentemente ou sempre. A pontuação mínima de 26 pontos e máxima de 130. A análise fatorial demonstrou através do alfa de Cronbach valores para os fatores: exploração do ambiente (alfa 0,89 ) 
e exploração de si (alfa 0,85 ) obtendo desta forma, bons índices de consistência interna.

\subsection{Procedimentos de coleta de dados}

Após a autorização do Comitê de Ética da Universidade São Francisco e dos coordenadores de cada curso, foram combinados os dias e horários da aplicação dos instrumentos com os professores. Em sala de aula foram explicados os objetivos da pesquisa aos estudantes, 0 caráter voluntário da participação e o sigilo de suas respostas e identidades. Posteriormente, os estudantes assinaram o Termo de Consentimento Livre e Esclarecido (TCLE), os instrumentos foram aplicados em sala de aula e de forma coletiva, levando em média 30 minutos.

\subsection{Procedimentos de análise dos dados}

Os dados foram organizados em uma planilha do SPSS (Statistical Package for the Social Sciences, versão 18.0) e submetidos à análise descritiva e inferencial, conforme os objetivos deste estudo. Para se analisar as relações entre os construtos recorreu-se à prova de correlação de Pearson e para verificar as diferenças foi utilizado o teste-t de Student para a comparação dos escores em razão da variável sexo e à análise de variância (ANOVA) para idade e o curso universitário.

\section{Resultados}

Os resultados seguirão a ordem dos objetivos, assim são apresentadas na Tabela 1 as correlações entre a Escala de Vida Acadêmica (EAVA) e a Escala de Exploração Vocacional (EEV).

Tabela 1

Coeficientes de correlação de Pearson entre os fatores da EAVA e as subescalas da EEV.

\begin{tabular}{lccc}
\hline Fatores da EAVA & & $\begin{array}{c}\text { Exploração do } \\
\text { ambiente }\end{array}$ & $\begin{array}{c}\text { Exploração } \\
\text { de si }\end{array}$ \\
\hline Ambiente universitário & $r$ & 0,10 & 0,04 \\
$\begin{array}{l}\text { Compromisso com o curso } \\
\text { Habilidade do estudante }\end{array}$ & $r$ & $0,19(* *)$ & $0,18\left(^{* *}\right)$ \\
$\begin{array}{l}\text { Envolvimento em atividades não } \\
\text { obrigatórias }\end{array}$ & $r$ & $0,30(* *)$ & $0,21\left(^{* *}\right)$ \\
$\begin{array}{l}\text { Condições para o estudo e } \\
\text { desempenho acadêmico }\end{array}$ & $r$ & 0,12 & $0,14(* *)$ \\
\hline$* * p<0,01$ & $r$ & 0,06 & $-0,02$ \\
\hline
\end{tabular}

$* * p<0,01$ 
Verifica-se na Tabela 1 que os fatores da EAVA, compromisso com o curso e habilidade do estudante apresentaram correlações significativas e positivas, de magnitude baixa, com as subescalas da EEV, exploração do ambiente e exploração de si. Ainda, o fator envolvimento em atividades não-obrigatórias da EAVA apresentou correlação positiva e estatisticamente significativa com a subescala exploração de si da EEV.

Procurou-se, também, analisar possíveis diferenças entre a amostra estudada. Os resultados para a variável sexo são apresentados na Tabela 2.

Tabela 2

Estatística descritiva e níveis de significância (p) para os fatores da EAVA e as subescalas da EEV, considerando a variável sexo dos estudantes.

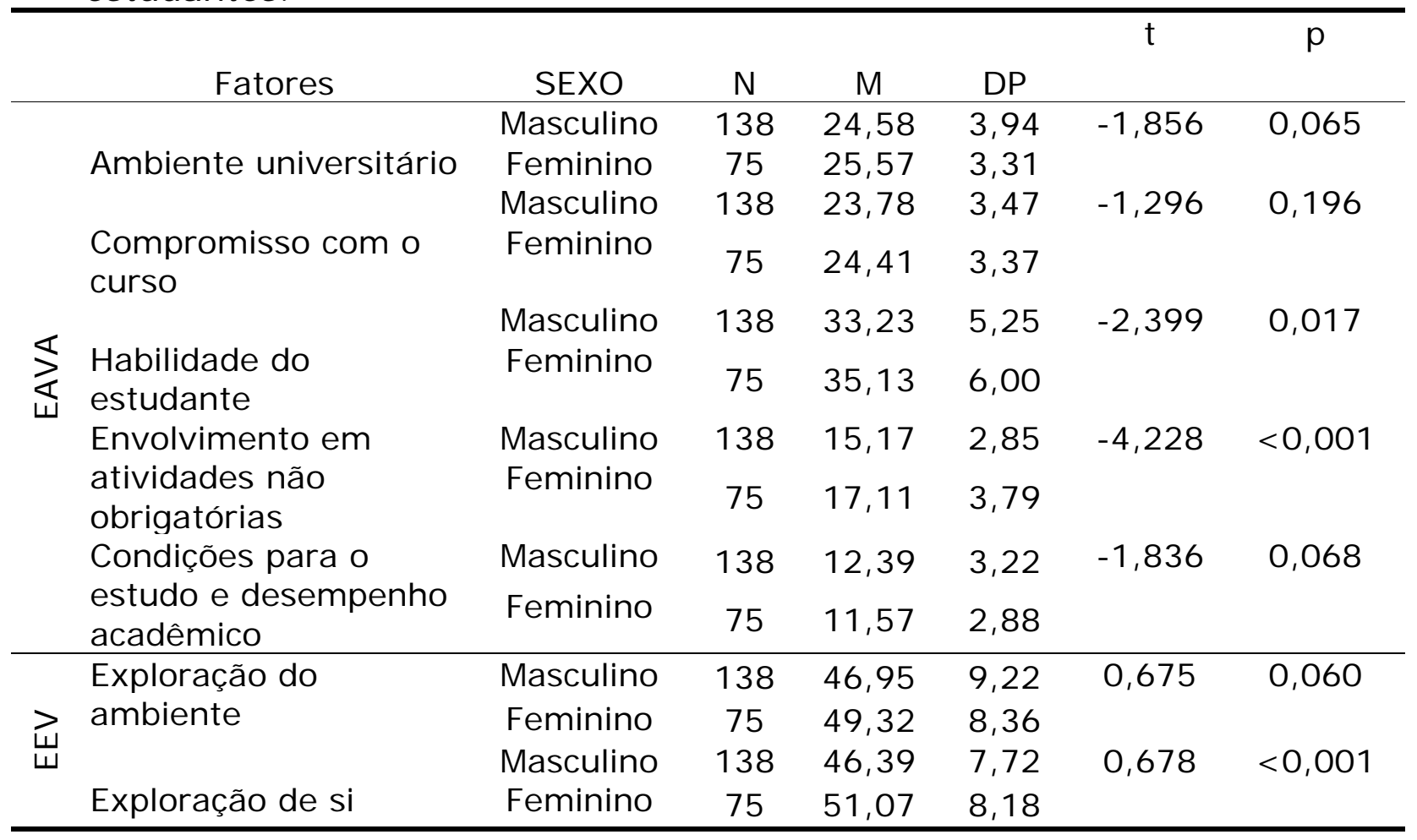

É possível observar na Tabela 2 que os homens obtiveram médias maiores que as das mulheres apenas no fator condições para o estudo e desempenho acadêmico, nos demais fatores da EAVA e os da EEV as mulheres obtiveram médias superiores. No entanto, foi aplicado o teste-t de Student e foram identificadas diferenças estatisticamente significativas apenas nos fatores habilidade do estudante e envolvimento em atividades não obrigatórias, em que as mulheres obtiveram pontuação superior à dos homens. A mesma análise foi feita com as dimensões da EEV e os resultados indicaram 
pontuação significativamente mais elevada para as mulheres somente na dimensão exploração de si.

Em relação à idade, os estudantes da faixa etária dos 21 a 23 anos obtiveram maiores médias em todos os fatores da EAVA que os demais grupos de idade. Ao aplicar a ANOVA foram encontradas diferenças significativas nos seguintes fatores da EAVA, compromisso com o curso $[F(3,209)=35,589 ; p=0,004]$; habilidade do estudante $[F(3,209)=108,742 ; p=0,026]$ e envolvimento em atividades não obrigatórias $[F(3,209)=38,512 ; p=0,002]$. Na EEV os estudantes obtiveram médias muito próximas em ambas às dimensões e a ANOVA indicou não haver diferenças estatisticamente significativas.

Para os cursos frequentados pelos universitários, foram identificadas médias superiores para os alunos do curso de Fisioterapia nos fatores da EAVA (ambiente universitário, compromisso com o curso, habilidade do estudante e envolvimento em atividades não obrigatórias) e ainda, na dimensão da EEV (exploração do ambiente). Por sua vez, os alunos do curso de Engenharia Civil alcançaram as maiores médias no fator da EAVA (condições para o estudo e desempenho acadêmico), enquanto que os alunos da Psicologia obtiveram pontuação maior na dimensão da EEV (exploração de si). Procurando diferenças estatisticamente significativas em relação aos cursos recorreu-se à ANOVA e foram constatadas nos fatores da EAVA, ambiente universitário $[F(5,206)=21,307 ; p=0,023]$; compromisso com o curso $[F(5,206)=13,403 ; p=0,004]$; habilidade do estudante $[F(5,206)=22,530 ; p=0,032]$ e envolvimento em atividades não obrigatórias $[F(5,206)=21,703 ; p=0,023]$.

A prova do post-hoc de Tukey indicou que, o curso de Fisioterapia obteve maiores médias que a Engenharia Mecânica no fator ambiente universitário. No fator compromisso com o curso também foi Fisioterapia que obteve maior média diferenciando-se dos alunos da Engenharia Elétrica; no fator habilidade do estudante os alunos do curso de Fisioterapia obtiveram maior média que os da Psicologia. Enquanto que no fator envolvimento em atividades não obrigatórias, também os alunos do curso de Fisioterapia obtiveram médias maiores diferenciando-se dos da Engenharia Mecânica.

Em relação à escala de exploração vocacional os alunos dos cursos de Fisioterapia, Psicologia e Engenharia da Computação obtiveram as maiores médias e muito próximas se comparadas aos outros cursos na dimensão exploração do ambiente, o mesmo ocorreu na dimensão exploração de si para os alunos do curso de Psicologia e Fisioterapia. Ao aplicar-se a ANOVA foram encontradas diferenças significativas somente para a dimensão exploração de si $[F(5,206)=41,696$; $p=0,038$. A prova post-hoc de Tukey permitiu que se identificasse que os alunos do curso de Psicologia obtiveram maiores médias que os da Engenharia Mecânica. 


\section{Discussão}

A vida acadêmica universitária está associada a diversos aspectos do desenvolvimento psicossocial, cognitivo, ajustamento à universidade, desempenho acadêmico e profissional (Almeida \& Soares, 2004; Bardagi, 2005; Teixeira et al., 2007). Os estudos que buscam compreender as variáveis e construtos que estão relacionados à vida acadêmica têm se mostrado importantes diante do grande número de estudantes universitários e das transformações sociais, econômicas e culturais que a sociedade está passando (Santos et al., 2010).

Assim, o primeiro objetivo deste estudo foi analisar a relação entre os construtos vida acadêmica e a exploração vocacional. Os resultados indicaram correlações significativas entre os fatores da EAVA (compromisso com o curso e habilidade do estudante) com as dimensões da EEV (exploração do ambiente e exploração de si), indicando que os alunos com mais certeza em relação ao curso frequentado, com maior percepção pessoal de suas habilidades apresentam maior autoconhecimento sobre si e são os que buscam mais informações sobre o mercado de trabalho. Assim, infere-se que os estudantes interessados no desenvolvimento de sua profissão busquem mais informações sobre as suas habilidades e também as necessidades requeridas pelo mercado de trabalho, estabelecendo metas mais realistas para a sua carreira. Como verificado nas pesquisas de Bardagi e Boff (2010) e Bardagi e Hutz (2010) que os alunos que têm planos definidos para o futuro apresentam índices maiores de autoeficácia, comportamento exploratório e comprometimento com a carreira, o que os fortalece para lidar com as peculiaridades do mercado de trabalho além de assumirem maior responsabilidade com sua inserção profissional.

O fator envolvimento em atividades não obrigatórias (EAVA) relacionou-se de forma positiva e significativa com a dimensão exploração de si (EEV). Assim, pode-se afirmar que as atividades que não se configuram como exigência formal do curso, mas que são promovidas ou incentivadas pela universidade contribuem para 0 autoconhecimento das preferências e das habilidades dos estudantes. Esses dados são congruentes como os verificados por Teixeira e Gomes (2004) que encontraram no seu estudo que os estudantes mais envolvidos em práticas não obrigatórias eram justamente os que tinham projetos pós-universidade já formulados. Dessa forma, os autores relatam resultados positivos para o desenvolvimento vocacional e pessoal quando as atividades não obrigatórias são relacionadas à área de formação. Vale ressaltar que não apenas as atividades não obrigatórias que se relacionam com os comportamentos de exploração vocacional, pois no estudo Bardagi e Hutz (2010) verificou-se que o envolvimento nas atividades 
acadêmicas, oferecidas pelas universidades, também se relaciona com a exploração de si e do ambiente.

Quanto ao estudo das variáveis sociodemográficas, os resultados da presente pesquisa mostraram diferenças significativas com maiores médias para as mulheres nos fatores da EAVA - habilidade do estudante e envolvimento em atividades não obrigatórias e na dimensão exploração de si da EEV. Assim, verificou-se que as mulheres pesquisadas relataram maior habilidade de resolução de problemas, maior envolvimento em atividades não obrigatórias e maior autoconhecimento sobre si e de suas habilidades. No estudo de Santos, Mognon, Lima et al. (2011) também foram encontradas diferenças significativas na EAVA com maior média para as mulheres, entretanto, foram nos fatores compromisso com o curso, condições para o estudo e desempenho acadêmico. Quanto a EEV, resultado favorável também foi encontrado para as mulheres na pesquisa de Bardagi e Boff (2010), mas diferentemente foi na dimensão exploração do ambiente e para o total. Entretanto, outros estudos mostram homogeneidade indicando não haver diferenças para a exploração vocacional quando considerada a variável sexo (Bardagi \& Hutz, 2010; Teixeira et al., 2007).

Para as idades, as diferenças estatisticamente significativas apareceram somente na EAVA, nos fatores compromisso com o curso, habilidade do estudante e envolvimento em atividades não obrigatórias com maiores médias para os estudantes da faixa etária dos 21 a 23 anos. Assim infere-se que os estudantes considerados 'tradicionais', ou seja, aqueles estudantes que entraram na universidade logo após terminarem os seus estudos no Ensino Médio, declararam maior certeza quanto à escolha do curso e maior percepção de segurança quanto à sua formação, tendendo a melhores habilidades básicas e maior engajamento na busca por experiências de enriquecimento profissional além de sua formação básica acadêmica. Apesar de Lemos (2010) ter separado de forma diferente as faixas etárias, quando comparados ao presente trabalho, os resultados apresentam certas semelhanças, principalmente nos fatores ambiente universitário e envolvimento em atividades não obrigatórias. Enquanto que no estudo de Santos, Mognon, Lima et al. (2011) não foram encontradas diferenças estatisticamente significativas para a idade. Diante dessas semelhanças e diferenças para a variável idade na EAVA, considera-se a necessidade de novos estudos.

Em relação aos cursos, os estudantes de Fisioterapia obtiveram pontuações mais elevadas e significativas nos fatores ambiente universitário, compromisso com o curso, habilidade do estudante e envolvimento em atividades não obrigatórias da EAVA. Isso significa dizer, que os alunos deste curso tendem a ter maior conhecimento sobre normas, programas, currículo acadêmico e serviços oferecidos 
pela universidade. Conjuntamente apresentam melhor percepção de suas competências cognitivas e maior envolvimento em atividades não obrigatórias a fim de aprimorarem sua formação com experiências acadêmicas, científicas, sociais, culturais e esportivas (Vendramini et al.,2004). Diante de tais resultados pode-se hipotetizar que algo específico relacionado ao curso pode ter interferido em tais resultados, como o interesse pessoal dos próprios alunos, os relacionamentos interpessoais com professores, coordenador e colegas, além de aspectos do próprio ambiente universitário como organização curricular, espaço físico, atividades oferecidas neste curso, importância social do curso e da instituição, dentre outros. Segundo Almeida e Soares (2004) algumas dessas variáveis são importantes para o sentimento de realização acadêmica nos estudantes.

No tocante ainda aos cursos, os estudantes de Psicologia apresentaram resultados estatisticamente significativos em exploração de si da EEV, o qual envolve maior reflexão sobre as próprias características de personalidade, interesses, habilidades, valores e influências relacionadas à escolha ocupacional. Pode-se inicialmente imaginar que a reflexão sobre si mesmo seja uma característica incentivada neste curso, entretanto, este resultado difere do encontrado na pesquisa de Gerk et al. (2011) que constataram que os alunos do curso de Psicologia obtiveram menores escores médios na dimensão exploração de si do que os estudantes do curso de Direito. Assim, parece que para se afirmar que tais aspectos acadêmicos podem ser influenciados pelo curso ainda precisam de mais estudos.

\section{Considerações finais}

Atualmente, a universidade tem sido vista como uma das possibilidades para a inserção no mercado de trabalho. Com isso, espera-se que o espaço universitário proporcione além da aquisição de novos conhecimentos, também nova visão sobre a profissão escolhida, o contato com novos valores, experiências acadêmicas e sociais que proporcionem ao estudante o amadurecimento pessoal e o impulsionem profissionalmente.

Um dos objetivos desta pesquisa foi estudar os aspectos da adaptação acadêmica e exploração vocacional no momento de conclusão do curso, o que vem sendo apresentado por muitos estudantes como um momento de grandes expectativas. Os resultados, como já hipotetizados, indicaram que aspectos pessoais do estudante como a satisfação e certeza na escolha do curso, bem como maior percepção de suas habilidades relacionam-se com a 
exploração vocacional, tanto de suas habilidades como do ambiente profissional.

Vale ressaltar que, atualmente, já se sabe que as próprias experiências acadêmicas favorecem o amadurecimento pessoal e profissional nos estudantes. No entanto, a contribuição das universidades pode ser maior quando os conteúdos e práticas oferecidas aos alunos são condizentes com o mercado de trabalho. Sugere-se assim, o desenvolvimento de experiências a fim de aumentar a exploração vocacional como, por exemplo, semanas de estudos, palestras, experiências práticas e o incentivo aos estágios para que os alunos aprimorem as suas habilidades e contribuam na formação da crença de que são capazes de arrumar um emprego após a formatura.

O contexto universitário e tudo o que circunda o estudante é importante ao se avaliar a vida acadêmica. Na presente pesquisa foram investigadas as possíveis diferenças para sexo, idade e cursos. No que se refere a primeira variável, as mulheres demonstraram acreditar mais no seu potencial, bem como procuram mais por atividades que vão além das exigência formais do curso e apresentam maior autoconhecimento. De modo geral, os estudos encontrados na literatura diferem dos resultados encontrados na presente pesquisa quanto a variável sexo tanto para a EAVA como para a EEV. Assim, os estudos precisam continuar a fim de verificar quais aspectos podem estar relacionadas à vida acadêmica e à melhor preparação para o mundo do trabalho. Essa compreensão poderá ampliar a visão dos fenômenos que ocorrem na universidade e contribuir para a criação de projetos que possam auxiliar o estudante a integrar-se à instituição de ensino e incrementar sua formação pessoal e profissional.

Para a faixa etária, os resultados indicaram diferenças significativas somente na EAVA em que os estudantes da faixa etária dos 21 a 23 anos declararam maior certeza quanto à escolha do curso e segurança na sua formação, além de maior engajamento na busca por atividades não-obrigatórias. Os resultados para essa variável também se mostraram controversos, tendo um estudo que não encontrou diferença significativa e outro que verificou diferenças significativas em diferentes fatores da EAVA. No entanto, nos três estudos os grupos de idade foram separados diferentemente o que pode ter interferido nos resultados. Assim, são necessários novos estudos, bem como a necessidade de que a separação por faixa etária seja realizada de maneiras similares, a fim de possibilitar comparações com os achados de outras pesquisas e possíveis generalizações.

Quanto à comparação entre os cursos frequentados pelos estudantes, os alunos de Fisioterapia apresentaram resultados superiores em diversos fatores da escala de vida acadêmica, o que sugere que 
apresentaram maior adaptação à universidade. Hipotetiza-se que tais resultados podem ter sido possibilitados pela estrutura do curso, a grade horária, os professores, os eventos promovidos para os estudantes e outros fatores pertinentes. Seria interessante promover um estudo qualitativo com essa amostra para verificar quais são as variáveis que favoreceram tais resultados o que podem vir a contribuir na elaboração de medidas de intervenção para outros cursos e instituições de ensino.

Apesar das limitações deste estudo, especialmente em razão do tamanho da amostra, do fato de ser proveniente de uma única IES e de diversos cursos, espera-se que os dados encontrados possam juntar-se a outros, resultantes da preocupação em compreender o momento de finalização do curso, e somem-se à ideia de que ações regulares das universidades devem ser propostas para os alunos e, em especial, para os formandos. Os dados obtidos mostram a relevância do papel das IES na adaptação acadêmica dos estudantes e na abertura a possibilidades de exploração vocacional com perspectivas de futura inserção e satisfação profissional. Independentemente de eventuais modificações que venham a ser introduzidas nos cursos, seria recomendável que projetos de intervenção no contexto acadêmico abordassem aspectos que são muito importantes para o desenvolvimento dos alunos nessa etapa educacional, dentre eles, escolha adequada do curso, dificuldades de aprendizagem, organização dos estudos e do tempo, habilidades acadêmicas e profissionais, oportunidades de atividades não obrigatórias, informações sobre a sua profissão no mercado como aspectos de inserção, salários e especializações.

\section{Referências}

Almeida, L. S., Soares, A. P. \& Ferreira, J. A. G. (2002). Questionário de vivência acadêmicas (QVA-r): avaliação do ajustamento dos estudantes universitários. Avaliação Psicológica, 1(2), 81-93.

Almeida, L. S. \& Soares, A. P. (2004). Os estudantes universitários: sucesso escolar e desenvolvimento psicossocial. In E. Mercuri \& S. A. J. Polydro (Eds). Estudante universitário: características e experiências de formação (pp. 15-41). São Paulo: Cabral.

Bardagi, M. P. (2005). A experiência universitária e a evasão no ensino superior: aspectos contextuais e vocacionais. Tese de Doutorado, Universidade Federal do Rio Grande do Sul, Porto Alegre. Bardagi, M. P. \& Boff, R. M. (2010). Autoconceito, autoeficácia profissional e comportamento exploratório em universitários. Revista Avaliação, 15(1), 41-56.

Bardagi, M. P. \& Hutz, C. S. (2010). Satisfação de vida, comprometimento com a carreira e exploração vocacional em 
estudantes universitários. Arquivos Brasileiros de Psicologia, 62(1), 159-170.

Gerk, E., Cardoso, J. A. R. \& Krafft, L. M. (2011). Ajustamento de alunos ingressantes ao ensino superior: o papel do comportamento exploratório vocacional. Revista Estudos e Pesquisa em Psicologia, 11(2), 719-724.

Granado, J. I. F. (2004). Vivência acadêmica de universitários brasileiros: estudo de validade e precisão do QVA-r. Dissertação de Mestrado, Universidade São Francisco, Itatiba.

Hoskins, S. L., Newstead, S. E., \& Dennis, I. (1997). Degree performance as a function of age, gender, prior qualifications and discipline studied. Assessment and Evaluation in Higher Education, 22(3), 317-328.

Lemos, T. H. (2010.) Escala de avaliação da vida acadêmica: estudo de validade com universitários da Paraíba. Dissertação de Mestrado, Universidade São Francisco, I tatiba.

Pelissoni, A. M. (2007). Autoeficácia na transição para o trabalho e comportamento de exploração de carreira em licenciados. Dissertação de Mestrado, Universidade Estadual de Campinas, Campinas.

Polydoro, A. J., Primi, R., Serpa, M. N. F., Zaroni, M. H. \& Pombal, K.C.P. (2001). Desenvolvimento de uma escala de integração ao Ensino Superior. Psico-USF, 6(1), 11-17.

Santos, A. A. A., Noronha, A. P., Amaro, C.B. \& Villar, J. (2005). Questionário de Vivência acadêmica: estudo de consistência interna do instrumento no contexto brasileiro. In M. C. R. A. Joly \& A. A. A. Santos, \& F. F. Sisto (Eds), Questões do cotidiano universitário. São Paulo: Casa do Psicólogo, p. 159-169.

Santos, A. A. A., Polydoro, S. A. J., Teixeira, M. A. \& Bardagi, M. P. (2010). Avaliação da integração do aluno ao ensino superior brasileiro. In A. A. A. Santos, F. F. Sisto, E. Boruchovitch \& E. Nascimento (Eds.). Perspectivas em avaliação psicológica (pp. 165188). São Paulo: Casa do Psicólogo.

Santos, A. A. A., Mognon, J. F. \& Joly, M. C. R. A. (2011). Crenças de autoeficácia na transição para o trabalho em formandos de Engenharia. Revista de Orientação Profissional, 12(2), 197-204.

Santos, A. A. A., Mognon, J. F., Lima, T. H. \& Cunha, N. B. (2011). A relação entre vida acadêmica e a motivação para aprender em universitários. Revista Psicologia Escolar e Educacional, 15(2), 283290.

Teixeira, M. A. P. \& Gomes, W. (2004). Estou me formando e agora? Reflexões e perspectivas de jovens formandos universitários. Revista Brasileira de Orientação Profissional, 5(1), 47-62.

Teixeira, M. A. P., Bardagi, M. P. \& Hutz, C. S. (2007). Escalas de exploração vocacional (EEV) para universitários. Psicologia em Estudo, 12(1), 175-182. 
Teixeira, M. A. P., Dias, A. C. G., Wotrich, S. H. E. \& Oliveira, A. M. (2008). Adaptação à universidade em jovens calouros. Psicologia Escolar e Educacional, 12(1), 185-202.

Trueman, M. \& Hartley, J. (1996). A comparison between the timemanagement skills and academic performance of mature and traditional-entry university students. Higher Education, 32 (2), 199215.

Vendramini, C. M. M., Santos, A. A. A., Polydoro, S. A. J., Sbardelini, E. T. B. Serpa, M. N. F. \& Natário, E. G. (2004). Construção e validação de uma escala de avaliação da vida acadêmica. Estudos de Psicologia, 9(2), 259-268.

Vieira, D. \& Coimbra, J. L. (2006). A autoeficácia na transição para o trabalho. In R. G. Azzi \& S. A. Polydoro (Eds.), Autoeficácia em diferentes contextos (pp. 25-58). Campinas: Átomo \& Alínea.

\section{Endereço para correspondência Jocemara Ferreira Mognon}

Universidade São Francisco - Programa de Pós-Graduação em Psicologia Rua Alexandre Rodrigues Barbosa, 45, CEP 13251-040, Itatiba - SP, Brasil. Endereço eletrônico: jocemognon@gmail.com

\section{Acácia A. Angeli dos Santos}

Universidade São Francisco - Programa de Pós-Graduação em Psicologia Rua Alexandre Rodrigues Barbosa, 45, CEP 13251-040, Itatiba - SP, Brasil. Endereço eletrônico: acacia.angeli@gmail.com

Recebido em: 25/06/2012

Reformulado em: 10/10/2013

Aceito para publicação em: 21/10/2013

Acompanhamento do processo editorial: Deise Maria Leal Fernandes Mendes

\section{Notas}

As autoras agradecem o financiamento da pesquisa de iniciação científica oferecido pelo CNPq à primeira autora.

* Psicóloga. Mestre e doutoranda em Psicologia na área de concentração em Avaliação Psicológica na Universidade São Francisco-Itatiba/SP e atualmente bolsista CAPES.

** Psicóloga. Doutora em Psicologia Escolar e Desenvolvimento Humano pela USP e Docente da Graduação e do Programa de Pós-Gradução Stricto Sensu em Psicologia da Universidade São Francisco - Itatiba/SP. 